\title{
A signal detection analysis of contingency data
}

\author{
LORRAINE G. ALLAN, SHEPARD SIEGEL, and JASON M. TANGEN \\ McMaster University, Hamilton, Ontario, Canada
}

\begin{abstract}
There are many psychological tasks that involve the pairing of binary variables. The various tasks used often address different questions and are motivated by different theoretical issues and traditions. Upon closer examination, however, the tasks are remarkably similar in structure. In the present paper, we examine two such tasks, the contingency judgment task and the signal detection task, and we apply a signal detection analysis to contingency judgment data. We suggest that the signal detection analysis provides a novel interpretation of a well-established but poorly understood phenomenon of contingency judgments- the outcome-density effect.
\end{abstract}

We must often make a decision even though the information we have is ambiguous or uncertain. One such situation is illustrated by a patient being treated by an allergist. The patient sometimes, but not always, develops hives after eating strawberries. Moreover, the patient sometimes develops hives even when strawberries are not eaten. Although the relationship between eating strawberries and developing hives is uncertain, the allergist must decide whether or not to recommend that the patient stop eating strawberries. Another type of ambiguous situation is illustrated by the task confronted by the radiologist. The radiologist must decide whether or not an X-ray indicates the presence of lung cancer. The signals seen in the X-ray are ambiguous, some consistent with lung cancer and others inconsistent with lung cancer. Even though the correct diagnosis is unclear, the radiologist must decide whether or not to recommend treatment. Despite the obvious similarities between the tasks, they have been treated quite differently. The allergy task has often been used by researchers interested in contingency assessment; that is, how humans judge that a cue (strawberry ingestion) imperfectly signals an outcome (see Allan, 1993; Shanks, 1993; Shanks, Holyoak, \& Medin, 1996). The cancer task has often been used by researchers interested in signal detection; that is, how humans make decisions about the presence of a signal (cancer symptoms) in a noisy background (see Green \& Swets, 1966; Macmillan \& Creelman, 1991; Swets, 1996; Wickens, 2002).

The contingency assessment situation and the signal detection situation are similar in that the information on

The preparation of this paper was supported by research grants from the Natural Sciences and Engineering Research Council of Canada to L.G.A. and S.S., by a grant from the U.S. National Institute on Drug Abuse to S.S., and by a Natural Sciences and Engineering Research Council of Canada Graduate Scholarship to J.M.T. We acknowledge the help provided by Thomas Wickens with regard to fitting ROCs to our data, and we thank Ralph Miller and John Wixted for their helpful comments on an earlier version of this paper. Correspondence should be addressed to L. G. Allan or S. Siegel, Department of Psychology, McMaster University, Hamilton, ON, L8S 4K1, Canada (e-mail: allan@mcmaster.ca or siegel@mcmaster.ca). which the decision is based is uncertain. Yet research concerned with contingency assessment and research concerned with signal detection have progressed independently, each with its own traditions and each motivated by different theoretical perspectives and models. In the present paper, we integrate these two lines of research. We suggest that contingency assessment is a form of signal detection. We demonstrate the value of this approach by providing a signal detection interpretation of a wellestablished but poorly understood phenomenon of contingency judgments - the outcome-density effect. Although there have been many demonstrations of the outcomedensity effect, we will limit ourselves to those seen in the "trial version" of the contingency assessment task; that is, tasks in which values of two binary variables, cues and outcomes, occur on each trial, and the observer must judge the strength of the relationship between them.

\section{The Contingency Assessment Task}

On each trial, the cue either is presented (C) or is not presented $(\sim C)$, and then the outcome either does occur (O) or does not occur $(\sim \mathrm{O})$. For example, in the previously described allergy task, the patient consumed or did not consume strawberries, and then the allergic reaction occurred or did not occur. After a series of trials on which each of the four cue-outcome combinations is presented with a predefined probability, the observer is asked to rate the strength of the relationship between the cue and the outcome. Table $1 \mathrm{~A}$ presents the $2 \times 2$ matrix for the cue-outcome pairings. The letters in the cells (A, B, C, D) represent the joint frequency of occurrence of the four cue-outcome combinations in a block of trials. One measure of the contingency between the cue and the outcome is $\Delta P_{\mathrm{O}}$ (Allan, 1980):

$$
\Delta P_{\mathrm{O}}=P(\mathrm{O} \mid \mathrm{C})-P(\mathrm{O} \mid \sim \mathrm{C})=\frac{\mathrm{A}}{\mathrm{A}+\mathrm{B}}-\frac{\mathrm{C}}{\mathrm{C}+\mathrm{D}} .
$$

Many early studies of contingency assessment concentrated on exploring the observer's ratings of the contingency between the two binary variables and the actual contingency. While these studies often reported a high 
Table 1A

The $2 \times 2$ Matrix for the Cue-Outcome Pairings in the Contingency Task

\begin{tabular}{rrrr}
\hline & $\mathrm{O}$ & $\sim \mathrm{O}$ \\
\hline $\mathrm{C}$ & $\mathrm{A}$ & $\mathrm{B}$ \\
$\mathrm{C}$ & $\mathrm{C}$ & $\mathrm{D}$ \\
\hline
\end{tabular}

Note-The letters in the cells (A, B, C, D) represent the joint frequency of occurrence of the four cue-outcome combinations in a block of trials.

Table 1B

The $2 \times 2$ Matrix for the Cue-Prediction Pairings in the Contingency Task

\begin{tabular}{rrr}
\hline & $\mathrm{Y}$ & $\sim \mathrm{Y}$ \\
\hline $\mathrm{C}$ & $\mathrm{A}$ & $\mathrm{B}$ \\
$\sim \mathrm{C}$ & $\mathrm{C}$ & $\mathrm{D}$ \\
\hline
\end{tabular}

Note-The letters in the cells (A, B, C, D) represent the joint frequency of occurrence of the four cue-prediction combinations in a block of trials.

Table 1C

The $2 \times 2$ Matrix for the Signal-Feedback Pairings in a Detection Task

\begin{tabular}{rrrr}
\multicolumn{3}{c}{ Detection Task } \\
\hline $\mathrm{C}$ & $\mathrm{O}$ & $\sim \mathrm{O}$ \\
\hline $\mathrm{C}$ & $\mathrm{A}$ & $\mathrm{B}$ \\
$\mathrm{C}$ & $\mathrm{D}$ \\
\hline
\end{tabular}

Note-The letters in the cells (A, B, C, D) represent the joint frequency of occurrence of the four signal-feedback combinations in a block of trials.

Table 1D

The $2 \times 2$ Matrix for the Signal-Response Pairings in a Detection Task

\begin{tabular}{rrrr}
\hline & $\mathrm{Y}$ & $\sim \mathrm{Y}$ \\
\hline $\mathrm{C}$ & $\mathrm{A}$ & $\mathrm{B}$ \\
$\sim \mathrm{C}$ & $\mathrm{C}$ & $\mathrm{D}$ \\
\hline
\end{tabular}

Note-The letters in the cells (A, B, C, D) represent the joint frequency of occurrence of the four signal-response combinations in a block of trials.

correlation between rating judgment and $\Delta P_{\mathrm{O}}$, systematic departures from $\Delta P_{\mathrm{O}}$ were frequently noted (see Allan, 1993; Shanks, 1993; Shanks et al., 1996). One such departure has been termed the outcome-density effect.

\section{The Outcome-Density Effect}

For a fixed $\Delta P_{\mathrm{O}}$, ratings of contingency often are not constant but increase with the probability of the outcome, $P(\mathrm{O})$ :

$$
P(\mathrm{O})=\frac{\mathrm{A}+\mathrm{C}}{\mathrm{A}+\mathrm{B}+\mathrm{C}+\mathrm{D}} .
$$

Among the first demonstrations of the outcome-density effect was that of Allan and Jenkins (1983, Experiment 3). In the Allan and Jenkins experiment, observers were required to judge the influence of the movement of a computer-generated joystick on the movement of a dot. At the beginning of a trial, the joystick was represented in its resting position, on the left side of the computer screen, and the dot was presented in the middle of the right side of the screen. On each trial, the joystick was programmed either to move $(\mathrm{C})$ or to remain in its resting position $(\sim C)$, and then the dot either moved downward $(\mathrm{O})$ or remained stationary $(\sim \mathrm{O})$. At the end of a series of 50 cue-outcome pairings, the observer rated the influence of joystick position on dot movement on a 40-point scale. Allan and Jenkins varied the contingency between joystick movement and dot movement $\left(\Delta P_{\mathrm{O}}\right)$, the probability of dot movement $[P(\mathrm{O})]$, and the probability of joystick movement $[P(\mathrm{C})]$, resulting in 20 cueoutcome matrices (see Table 2). $P(\mathrm{C})$ was manipulated in this experiment because Allan and Jenkins were interested in evaluating a cue-density effect as well as the outcome-density effect. For 10 matrices, $P(\mathrm{C})=.5$, and for 10 matrices, $P(\mathrm{C})=.7$. There were 10 noncontingent $\left(\Delta P_{\mathrm{O}}=0\right)$ matrices and 10 contingent $\left(\Delta P_{\mathrm{O}}>0\right)$ matrices, and for a fixed value of $\Delta P_{\mathrm{O}}, P(\mathrm{O} \mid \mathrm{C})$ and $P(\mathrm{O} \mid \sim \mathrm{C})$ were varied. Each observer rated each of the 20 matrices that were presented in random order over two sessions. The data are replotted in Figure 1, which shows mean ratings for each of the 20 matrices. Ratings increased as $\Delta P_{\mathrm{O}}$ increased, but were also dependent on $P(\mathrm{O})$ - the outcome-density effect. Consider the 10 matrices where $\Delta P_{\mathrm{O}}=0$ (the leftmost 10 bars). As $P(\mathrm{O})$ increased from .1 to .9 , ratings also increased, even though $\Delta P_{\mathrm{O}}$ was constant. The same dependence on $P(\mathrm{O})$ is seen for the contingent matrices. Note also that ratings were higher when $P(\mathrm{C})=.7$ (the filled bars) than when $P(\mathrm{C})=.5$ (the open bars). That is, in addition to an outcome-density effect, Allan and Jenkins also found a cue-density effect.

In some contingency judgment experiments, observers have been asked to predict the outcome on each trial (e.g., Tangen \& Allan, 2003, 2004) in addition to rating the relationship between the cue and the outcome at the end of a block of trials. On each trial, the cue is either presented $(\mathrm{C})$ or is not presented $(\sim \mathrm{C})$, the observer predicts whether the outcome will be presented $(\mathrm{Y})$ or will not be

Table 2

Probabilities Used in Allan and Jenkins (1983, Experiment 3)

\begin{tabular}{|c|c|c|c|c|}
\hline$P(\mathrm{C})$ & $P(\mathrm{O} \mid \mathrm{C})$ & $P(\mathrm{O} \mid \sim \mathrm{C})$ & $P(\mathrm{O})$ & $\Delta P_{\mathrm{O}}$ \\
\hline \multirow[t]{10}{*}{.5} & .1 & .1 & .1 & 0 \\
\hline & .3 & .3 & .3 & 0 \\
\hline & .5 & .5 & .5 & 0 \\
\hline & .7 & .7 & .7 & 0 \\
\hline & .9 & .9 & .9 & 0 \\
\hline & .3 & .1 & .2 & .2 \\
\hline & .9 & .7 & .8 & .2 \\
\hline & .5 & .1 & .3 & .4 \\
\hline & .9 & .5 & .7 & .4 \\
\hline & .9 & .1 & .5 & .8 \\
\hline \multirow[t]{10}{*}{.7} & .1 & .1 & .1 & 0 \\
\hline & .3 & .3 & .3 & 0 \\
\hline & .5 & .5 & .5 & 0 \\
\hline & .7 & .7 & .7 & 0 \\
\hline & .9 & .9 & .9 & 0 \\
\hline & .3 & .1 & .24 & .2 \\
\hline & .9 & .7 & .84 & .2 \\
\hline & .5 & .1 & .38 & .4 \\
\hline & .9 & .5 & .78 & .4 \\
\hline & .9 & .1 & .66 & .8 \\
\hline
\end{tabular}




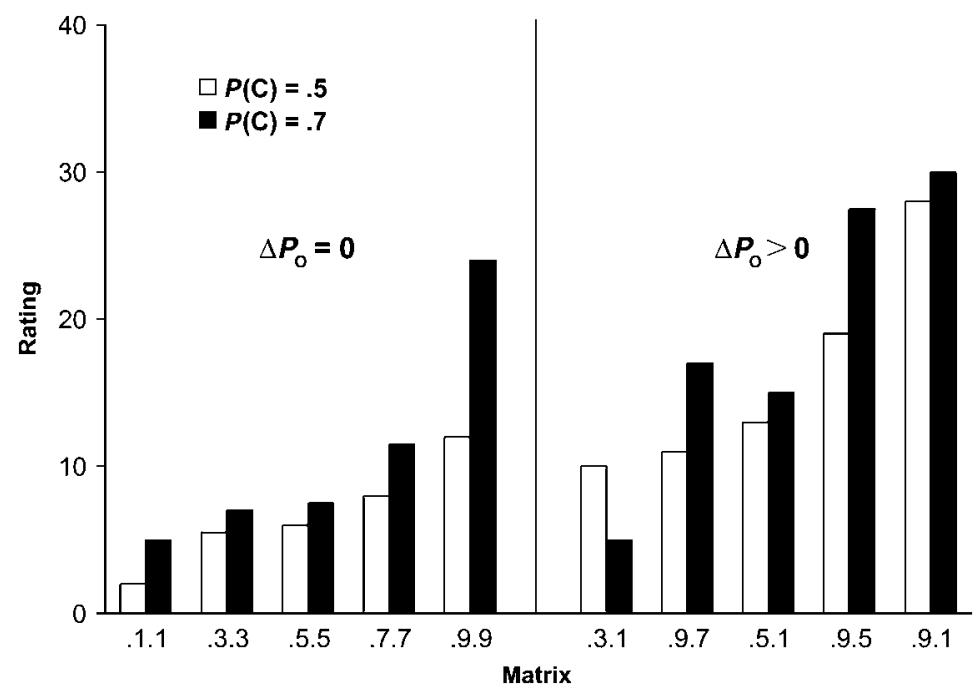

Figure 1. Ratings for the 20 matrices in Allan and Jenkins (1983, Experiment 3). The paired values on the abscissa are for $P(\mathrm{Y} \mid \mathrm{C})$ and $(\mathrm{Y} \mid \sim \mathrm{C})$. The data are replotted from the values in Allan and Jenkins (1983).

presented $(\sim \mathrm{Y})$, and then the outcome either occurs $(\mathrm{O})$ or does not occur $(\sim \mathrm{O})$. These trial-by-trial responses will hereafter be termed "prediction responses." Table 1B presents the $2 \times 2$ matrix for the cue-prediction pairings. The letters in the cells represent the joint frequency of occurrence of the four cue-prediction combinations in a block of trials. The contingency between the cue and the prediction response, $\Delta P_{\mathrm{PRED}}$, is

$$
\Delta P_{\mathrm{PRED}}=P(\mathrm{Y} \mid \mathrm{C})-P(\mathrm{Y} \mid \sim \mathrm{C})=\frac{\mathrm{A}}{\mathrm{A}+\mathrm{B}}-\frac{\mathrm{C}}{\mathrm{C}+\mathrm{D}} .
$$

Although prediction responses have been collected for years, they have rarely been analyzed or reported. Recently, some investigators have calculated $\Delta P_{\mathrm{PRED}}$ values and have reported a high correlation between $\Delta P_{\mathrm{PRED}}$ and $\Delta P_{\mathrm{O}}$ (e.g., Collins \& Shanks, 2002; Tangen \& Allan, $2003,2004)$. That is, the observer's trial prediction responses closely match the actual programmed contingency between the cue and the outcome. Moreover, there often appears to be a dissociation between $\Delta P_{\mathrm{PRED}}$ and the ratings given at the end of a block of trials.

As we noted earlier, there have been many studies that have reported density effects as measured by ratings. However, these studies either did not require trial prediction responses, or if they did, they did not report prediction data. The purpose of our experiment was to examine the effects of outcome density on ratings and on prediction responses. On the basis of previous data, we expected the ratings to show an outcome-density effect. We were interested in determining whether the prediction responses would also show an outcome-density effect, or whether there might be a dissociation between the two dependent measures. Moreover, as will subsequently be demonstrated, the prediction responses allow us to apply a signal detection analysis to our contingency assessment data.

\section{METHOD}

\section{Observers}

The observers were 40 undergraduate students enrolled in psychology courses at McMaster University who participated for course credit. They had not participated in other experiments concerned with contingency judgments.

\section{Apparatus}

Observers participated individually. Macintosh computers were used to present stimuli and record responses. The cues (chemicals) and outcomes (bacterial strains) were those used in our earlier research (e.g., Allan, Tangen, Wood, \& Shah, 2003). The presence of a cue (C) was indicated by a colored three-dimensional animation of a chemical spinning on its axis and the absence of a cue $(\sim \mathrm{C})$ was indicated by a faded stationary grayscale picture of the chemical. Similarly, the presence of the outcome $(\mathrm{O})$ was indicated by a colored animation of moving bacteria and the absence of the outcome $(\sim \mathrm{O})$ was indicated by a faded grayscale picture of stationary bacteria. There were four chemicals and four bacterial strains. For each observer, one chemical and one bacterial strain was used during the instructions and practice trials. Each observer participated in three experimental conditions, and the remaining three chemicals and three bacterial strains were randomly assigned to the three conditions. Each of the chemicals and strains was randomly assigned fictitious names. Each of the colored moving images (indicating the presence of cues and outcomes) was accompanied by the name (e.g., "Chorbine Present") in text presented on the computer screen. In contrast, the faded stationary images (indicating the absence of cues and outcomes) were not accompanied by text.

\section{Procedure}

The instructions for the experiment were presented to the observer on the computer monitor. In brief, the observer was told that scientists have recently discovered a number of strains of bacteria that exist in the mammalian digestive system. For each strain, the scientists were testing whether a chemical aids in, interferes with, or has no effect on a strain's survival. To do this, a strain of bacteria was first placed in culture (petri dishes). After that, a chemical might be added to the bacterial culture. The scientists then verified whether or not the bacterial sample survived. The observer was also 
shown the rating scale that the scientists would use to rate the effectiveness of the chemical on the survival of the bacteria. After reading the instructions, the observer was shown a summary screen of the four cue-outcome combinations using one of the chemicals and one of the bacterial strains. Eight practice trials were then presented in which each of the four cue-outcome combinations was presented twice in random order.

The six $2 \times 2$ matrices used in our experiment are shown in Table 3. For the three matrices on the left, $\Delta P_{\mathrm{O}}=0$, and for three matrices on the right, $\Delta P_{\mathrm{O}}=.467$. In each column, $P(\mathrm{O})$ increases from top to bottom. The two values of $\Delta P_{\mathrm{O}}(0$ and .467$)$ were varied between groups ( $n=20$ /group), and the three levels of $P(O)$ (low, medium, and high) were varied within groups. The order of the three $P(\mathrm{O})$ values was randomly determined for each observer.

The observer initiated a $P(\mathrm{O})$ condition by clicking on the "Begin" button on the computer screen, and was shown that the chemical was added or was not added. The observer then predicted whether or not the bacteria survived by clicking the appropriate button on the screen. Subsequently, the observer was shown whether or not the bacteria survived and was provided feedback (correct, incorrect) on his/her choice. The next trial was initiated by a mouse click on the "Next Trial" button.

In addition to prediction responses, the observer also provided rating responses. There were 60 trials and the observer rated how strongly the chemical affected the survival of the bacterial strain after Trials 20,40, and 60 . The ratings were made on a horizontal scrollbar that ranged from -100 (chemical has a very strong negative effect on the bacteria's survival) on the left to +100 (chemical has a very strong positive effect on the bacteria's survival) on the right, and was anchored at 0 in the middle. Observers made their ratings by moving the scrollbar left and right with the mouse.

\section{RESULTS}

Mean rating after the final (60th) trial for each outcome density is plotted for the two contingencies in Fig-

Table 3

The Matrices Used in the Experiment

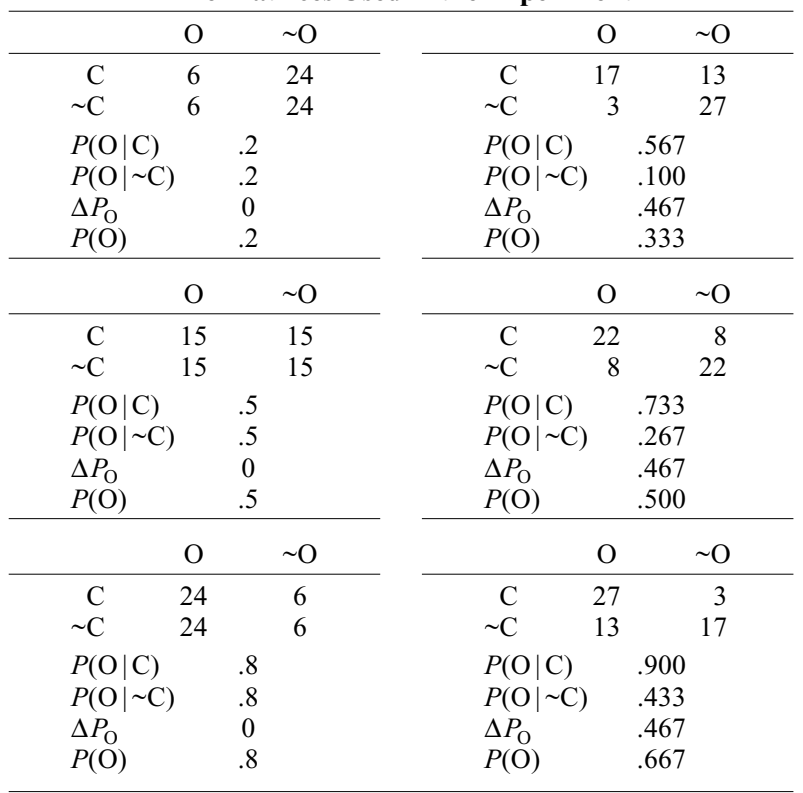

Note-For the three matrices on the left, $\Delta P_{\mathrm{O}}=0$, and for the three matrices on the right, $\Delta P_{\mathrm{O}}=.467$. In each column, $P(\mathrm{O})$ increases from top to bottom. ure 2, which clearly shows that ratings depend on both the actual contingency, $\Delta P_{\mathrm{O}}$, and the outcome density, $P(\mathrm{O})$. Overall the ratings are higher for the contingent matrices than for the noncontingent matrices, and for both contingencies, ratings increase with outcome density. That is, there is an outcome-density effect. The outcome-density effect is so pronounced that the rating of the high-density noncontingent matrix is larger than the ratings of the low- and medium-density contingent matrices.

Observers made three ratings for each matrix: one each after Trials 20, 40, and 60. Ratings as a function of trial are shown in Figure 3A for the noncontingent matrices and in Figure 3B for the contingent matrices. For the noncontingent matrices, the outcome-density effect is seen as early as Trial 20 . For the contingent matrices, the outcome-density effect is not evident at Trial 20 , but is by Trial 40 . A $2\left(\Delta P_{\mathrm{O}}: 0\right.$ and .467$) \times 3$ (outcome density: low, medium, high) $\times 3$ (trial: $20,40,60)$ mixed-design analysis of variance (ANOVA) conducted on the ratings confirmed the trends seen in Figure 3. Both the main effect of contingency and the main effect of density were significant $[F(1,38)=15.58, p<.001$, and $F(2,76)=$ $17.14, p<.001$, respectively]. The main effect of trial was not significant $[F(2,76)=2.08, p>.05]$, but trial did interact with contingency $[F(2,76)=4.34, p<.05]$.

Mean $\Delta P_{\mathrm{PRED}}$ values, based on all 60 trials, for each density are plotted for the two contingencies in Figure 4. In contrast to the ratings, $\Delta P_{\text {PRED }}$ does not vary systematically with outcome density. Rather, for both the noncontingent and the contingent matrices, the $\Delta P_{\mathrm{PRED}}$ values are similar across outcome density. A $2\left(\Delta P_{\mathrm{O}}: 0\right.$ and $.467) \times 3$ (density: low, medium, high) mixed-design ANOVA was conducted on $\Delta P_{\mathrm{PRED}}$ values. The main effect of contingency was significant $[F(1,38)=47.87$, $p<.001]$, but the main effect of density and the interaction between density and contingency were not significant $[F(2,76)=2.28, p>.05$, and $F(2,76)=.52, p>$ .05 , respectively].

$\Delta P_{\mathrm{PRED}}$ is the difference between two conditional probabilities (see Equation 3). An examination of these probabilities provides clarification regarding the relationship between outcome density and $\Delta P_{\mathrm{PRED}}$. Figure 5 plots $P(\mathrm{Y} \mid \mathrm{C}), P(\mathrm{Y} \mid \sim \mathrm{C})$, and $\Delta P_{\mathrm{PRED}}$ as a function of outcome density. The data for the three noncontingent matrices are seen in Figure 5A and the data for the three contingent matrices are seen in Figure 5B. For both sets of matrices, $P(\mathrm{Y} \mid \mathrm{C})$ and $P(\mathrm{Y} \mid \sim \mathrm{C})$ increase with outcome density. That is, as outcome density increases, so does the observer's tendency to predict that the outcome will occur, both on cue-present and on cue-absent trials. Although $P(\mathrm{Y})$ increases with $P(\mathrm{O})$, the $\Delta P_{\mathrm{PRED}}$ values are similar.

In summary, outcome-density affects the observer's tendency to predict that the outcome will occur $[P(\mathrm{Y})]$, but not the observer's sensitivity to the actual contingency $\left(\Delta P_{\text {PRED }}\right)$. Ratings of the contingency at the end of a block of trials show the same pattern of results as $P(\mathrm{Y})$ both increase with outcome density. We now elaborate a signal detection analysis of our data. 


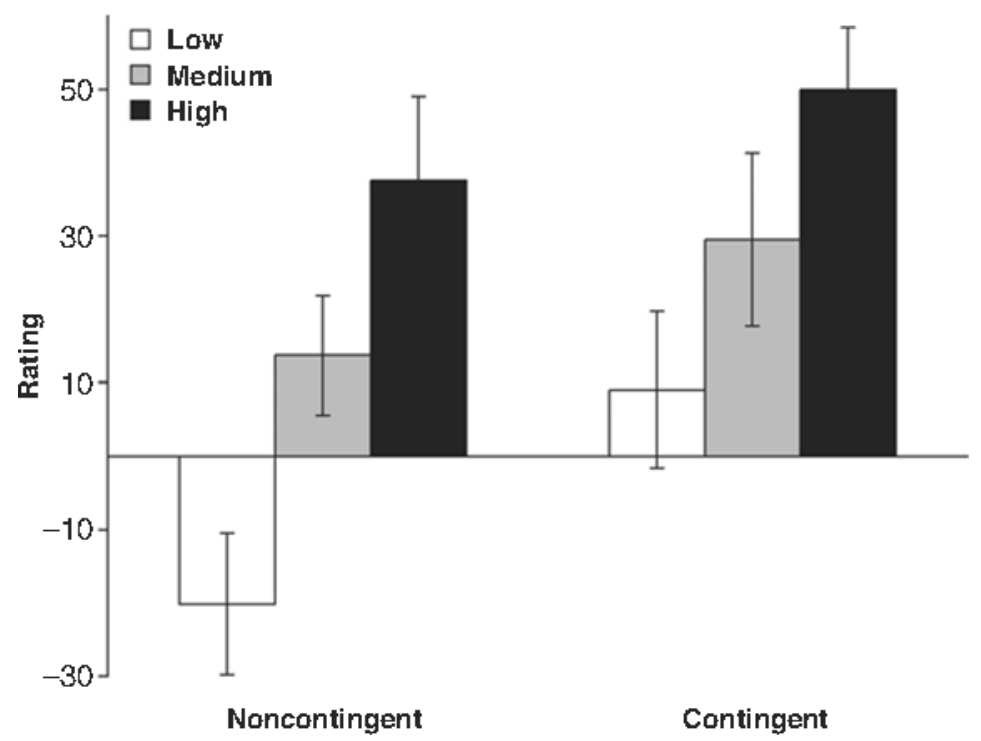

Figure 2. Ratings after the final (60th) trial for each outcome density (low, medium, and high). The ratings for the three noncontingent matrices $\left(\Delta P_{0}=0\right)$ are on the left, and the ratings for the three contingent matrices $\left(\Delta P_{O}=.467\right)$ are on the right.

\section{Signal Detection Theory (SDT)}

Early applications of SDT sought to account for decision making in simple psychophysical situations, such as detecting an auditory signal in a noisy background or a change in illumination in a visual background. As the value of the theory was established for understanding simple detection, its application expanded within psychophysics to more complex tasks such as discrimination and identification. SDT has been influential outside psychophysics as well, providing a conceptual framework for understanding many findings in diverse areas such as recognition memory, medical decision making, pigeon choice behavior, and the placebo effect (e.g., Allan \& Siegel, 2002; Blough, 2004; Swets, 1996).

For simplicity, we will first present SDT as applied to the simple signal detection task. In a detection task, on some trials the signal is not presented $(\sim \mathrm{C})$, and on the remaining trials the signal is presented (C). For example, in a tone detection task, on some trials only white noise is presented $(\sim \mathrm{C})$, and on the remaining trials a signal (e.g., a $1000-\mathrm{Hz}$ tone) is embedded in the noise (C). The observer's task is to indicate whether the signal was presented $(\mathrm{Y})$ or was not presented $(\sim \mathrm{Y})$. Often information (feedback) is provided to indicate whether the signal actually occurred $(\mathrm{O})$ or did not occur $(\sim \mathrm{O})$. Table $1 \mathrm{C}$ presents the $2 \times 2$ matrix for the signal-feedback pairings and Table 1D depicts the $2 \times 2$ matrix for the signalresponse pairings. As is readily apparent, Tables $1 \mathrm{~A}$ and 1B for the contingency assessment are the same as Tables $1 \mathrm{C}$ and $1 \mathrm{D}$ for the signal detection task. We suggest that the commonality in depiction reflects a commonality in mechanism.

Tables $1 \mathrm{~B}$ and $1 \mathrm{D}$ relate presented cues to responses made by the observer. In both cases, a cue is presented or not, and then the observer makes a response. For detection, the observer indicates whether the cue occurred or not; for contingency assessment, the observer predicts whether the cue will be followed by the outcome or not. For detection the response is about a cue that has occurred, whereas for contingency assessment the response is a prediction based on a cue that has occurred. Tables 1A and $1 \mathrm{C}$ relate presented cues to information provided by the experimenter. In both cases, a cue is presented or not, and then information about the cue is provided. For detection, the observer is told whether the cue occurred or not; for contingency assessment, the observer is told whether the cue was followed by the outcome or not. For detection the information is about the cue that might have occurred, whereas for contingency assessment the information is about whether the cue resulted in the outcome. Our goal is to show that these mappings, which treat contingency assessment as a form of signal detection, provide an informative analysis of our data.

The basic assumption underlying SDT is that repeated presentations of a constant physical stimulus do not result in a constant subjective value. Rather, the resulting subjective value is variable. This variability is the result of a number of factors, such as internal fluctuations in the observer's nervous system and external fluctuations in the environment. This inherent variability often can be approximated by the normal distribution and is referred to as noise. It is because a stimulus is always presented in a background of noise that the resulting subjective value is variable.

The observer's decision problem is represented schematically in Figure 6. The horizontal axis represents a random variable $X$ and the vertical axis represents the probability density of $X$ for the two stimuli $\sim \mathrm{C}$ and $\mathrm{C}-$ 

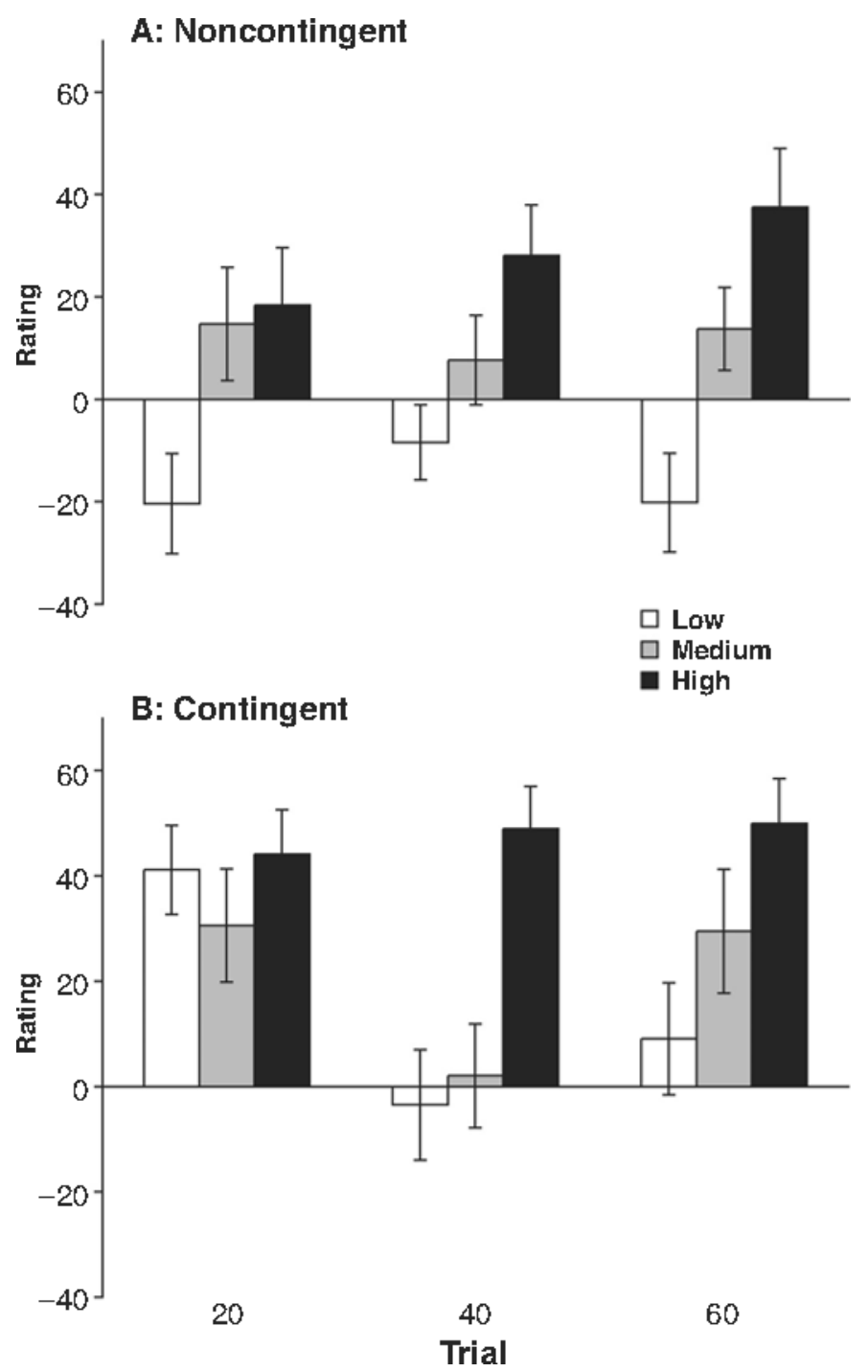

Figure 3. Ratings as a function of trial for each outcome density (low, medium, and high) for (A) noncontingent matrices and (B) contingent matrices.

that is, $f(X \mid \sim \mathrm{C})$ and $f(X \mid \mathrm{C})$, respectively. The random variable $X$ is often described as subjective values generated by the presented physical values; $f(X \mid \sim \mathrm{C})$ is the distribution of subjective values generated on $\sim \mathrm{C}$ trials (i.e., the noisy background) and $f(X \mid \mathrm{C})$ is the distribution generated on C trials. In the simplest version of SDT, it is assumed that the distributions are normal and differ only in mean. When the signal is weak, there will be considerable overlap of the $\sim \mathrm{C}$ and $\mathrm{C}$ distributions. The degree of overlap of the two distributions, $d^{\prime}$, is defined as the difference between the means of the two distributions normalized by their common standard deviation. Specifically,

$$
d^{\prime}=\frac{\mu_{\mathrm{C}}-\mu_{\sim \mathrm{C}}}{\sigma} .
$$

where $\mu_{\mathrm{C}}$ is the mean of the $\mathrm{C}$ distribution, $\mu_{\sim_{\mathrm{C}}}$ is the mean of the $\sim \mathrm{C}$ distribution, and $\sigma$ is the common standard devi- ation of the distributions. As the signal increases in strength relative to the noise, $\mu_{\mathrm{C}}$ will increase and so will $d^{\prime}$.

The observer's task is to decide whether it was more likely that the subjective value on a trial was generated by the signal or was due to noise. The observer solves this decision problem by setting a criterion value $\beta$ (Figure 6). If the subjective value is larger than $\beta$, the response is "signal" (Y), and if the subjective value is less than $\beta$, the response is "no signal" ( $\sim \mathrm{Y})$. The area to the right of $\beta$ under the $\mathrm{C}$ distribution in Figure 6 represents the probability that a subjective value larger than $\beta$ was generated by $\mathrm{C}$. An estimate of this probability is provided by $P(\mathrm{Y} \mid \mathrm{C})$, and the $z$-score associated with $P(\mathrm{Y} \mid \mathrm{C})$ provides an estimate of the placement of $\beta$ relative to $\mu_{\mathrm{C}}$ in $\sigma$ units,

$$
z(\mathrm{Y} \mid \mathrm{C})=\frac{\beta-\mu_{\mathrm{C}}}{\sigma} .
$$




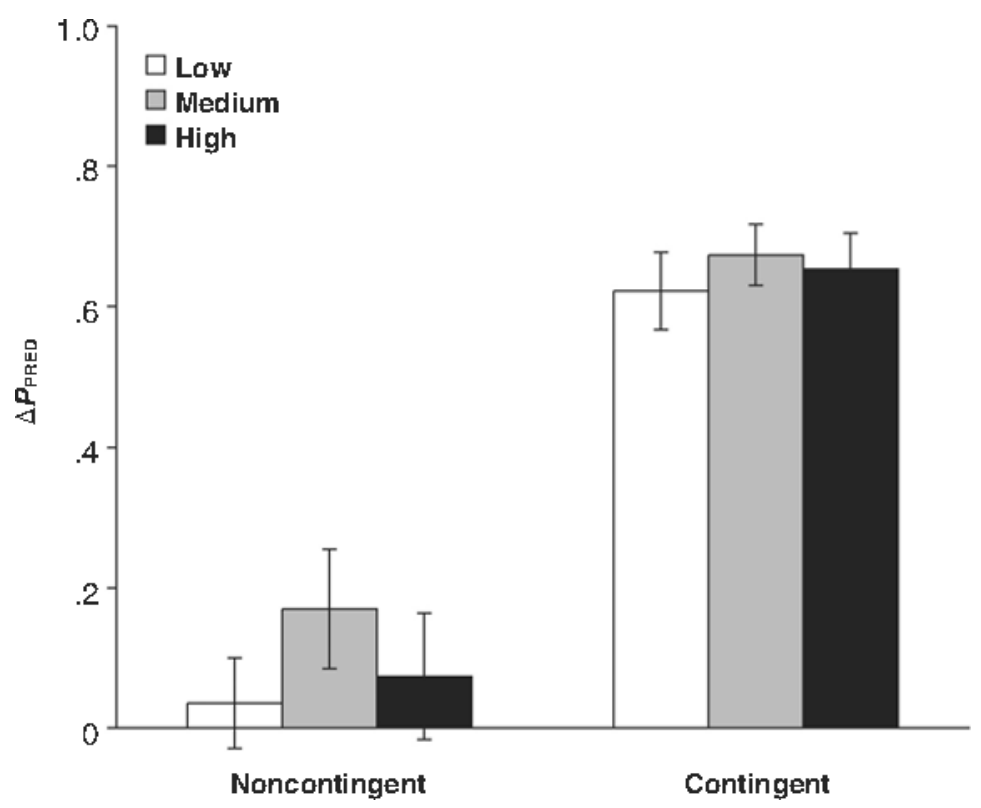

Figure 4. $\Delta P_{\text {PRED }}$, based on all 60 trials, for each outcome density (low, medium, and high). The values for the three noncontingent matrices $\left(\Delta P_{0}=0\right)$ are on the left, and the values for the three contingent matrices $\left(\Delta P_{O}=.467\right)$ are on the right.

The area to the right of $\beta$ under the $\sim \mathrm{C}$ distribution in Figure 6 represents the probability that a subjective value larger than $\beta$ was generated by $\sim \mathrm{C}$. An estimate of this probability is provided by $P(\mathrm{Y} \mid \sim \mathrm{C})$, and the $z$-score associated with $P(\mathrm{Y} \mid \sim \mathrm{C})$ provides an estimate of the placement of $\beta$ relative to $\mu_{\sim \mathrm{C}}$ in $\sigma$ units,

$$
z(\mathrm{Y} \mid \sim \mathrm{C})=\frac{\beta-\mu_{\sim \mathrm{C}}}{\sigma} .
$$

Combining Equations 5A and 5B yields

$$
z(\mathrm{Y} \mid \sim \mathrm{C})-z(\mathrm{Y} \mid \mathrm{C})=\frac{\mu_{\mathrm{C}}-\mu_{\sim \mathrm{C}}}{\sigma}=d^{\prime} .
$$

Note that Equation 6 specifies that $d^{\prime}$ estimated from the data is independent of the placement of $\beta$. Readers familiar with SDT will recognize that $P(\mathrm{Y} \mid \mathrm{C})$ is referred to as the probability of a hit, $P(\mathrm{H})$, and $P(\mathrm{Y} \mid \sim \mathrm{C})$ is referred to as the probability of a false alarm, $P(\mathrm{FA})$.

In Figure $6, \beta$ is located between the means of the two distributions. Figure 7 shows two other placements of $\beta$. If the observer is "liberal" about responding Y (Figure 7A), the criterion will be located below the mean of $\sim \mathrm{C}$ and $\mathrm{Y}$ will occur frequently. In contrast, if the observer is "conservative" about reporting Y (Figure 7B), the criterion will be located above the mean of $\mathrm{C}$ and $\mathrm{Y}$ will occur infrequently. Finally, if the observer adopts a moderate strategy, as in Figure 6, the criterion will be located between the two means, and the probability of $Y$ will be similar to the probability of $\sim$ Y. While the placement of $\beta$ influences $P(\mathrm{Y} \mid \mathrm{C})$ and $P(\mathrm{Y} \mid \sim \mathrm{C})$, it has no influence on $d^{\prime}$. To anticipate, we will demonstrate that the outcome-density effect results because outcome density affects the observer's response bias, $\beta$, and not the observer's sensitivity to $\Delta P_{\mathrm{O}}$.

Independence of $d^{\prime}$ and $\beta$ : The receiver operating characteristic (ROC). SDT provides for two independent indices that can be estimated from the observer's responses. The value of $d^{\prime}$ provides an estimate of the observer's sensitivity, and the value of $\beta$ provides an estimate of the observer's response criterion. This independence of $d^{\prime}$ and $\beta$ is best illustrated by what is referred to as the receiver operating characteristic (ROC). The axes of a ROC plot are $P(\mathrm{Y} \mid \mathrm{C})$ and $P(\mathrm{Y} \mid \sim \mathrm{C})$. Figure 8 plots three theoretical ROCs. For each ROC, the $\mathrm{C}$ and $\sim \mathrm{C}$ values are constant, and each point on the ROC is generated by a different location for $\beta$. As one moves along a specific ROC from the lower left corner [where $P(\mathrm{Y} \mid \mathrm{C})=P(\mathrm{Y} \mid \sim \mathrm{C})=0$ ] to the upper right corner [where $P(\mathrm{Y} \mid \mathrm{C})=P(\mathrm{Y} \mid \sim \mathrm{C})=$ $1.0]$, the criterion changes from maximally conservative to maximally liberal. The three ROCs in Figure 8 are generated by different signal values. As the signal strength increases, the ROC moves away from the diagonal. The three curves are for $d^{\prime}=1.0,1.5$, and 2.0.

An SDT Analysis of Contingency Data. Figure 9 plots $P(\mathrm{Y} \mid \mathrm{C})$ as a function of $P(\mathrm{Y} \mid \sim \mathrm{C})$ for the data from our experiment. The data are averaged over observers. The lower set of three points was generated from the three noncontingent matrices and the upper set of three points was generated from the three contingent matrices. Each curve is the best-fitting SDT ROC to the three data points. ${ }^{1}$ The fit assumes underlying normal distributions of equal variance. The deviations of the ROC from the data are not significant as measured by the Pearson test $\left[\chi^{2}(2)=\right.$ $.524, p>.05$ and $\chi^{2}(2)=.044, p>.05$, for the non- 


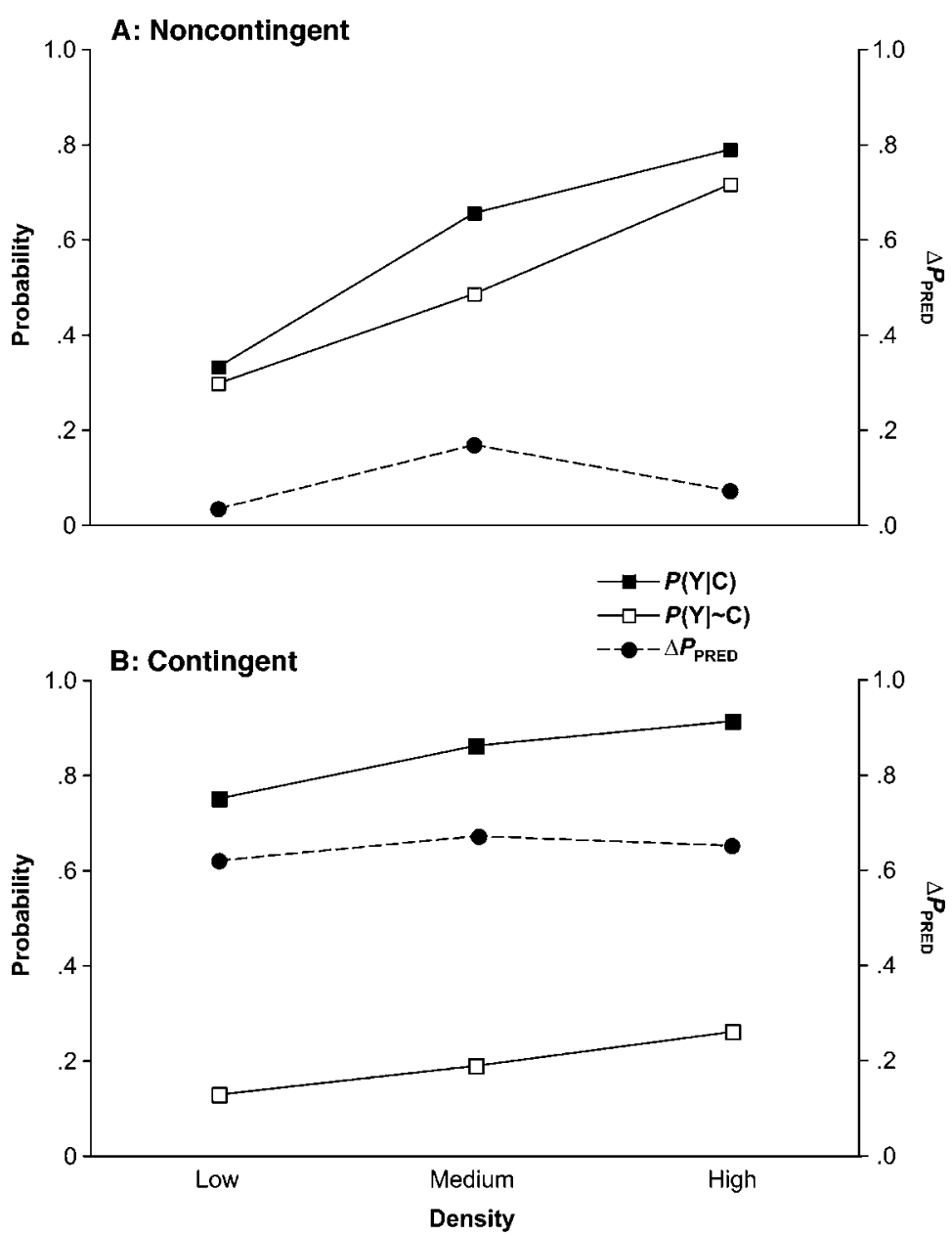

Figure 5. $P(\mathrm{Y} \mid \mathrm{C}), P(\mathrm{Y} \mid \sim \mathrm{C})$, and $\Delta P_{\mathrm{PRED}}$ as a function of outcome density. $P(\mathrm{Y} \mid \mathrm{C})$ and $P(\mathrm{Y} \mid \sim \mathrm{C})$ are plotted on the left ordinate, and $\Delta \boldsymbol{P}_{\mathrm{PRED}}$ is plotted on the right ordinate. (A) Noncontingent matrices; $(B)$ contingent matrices.

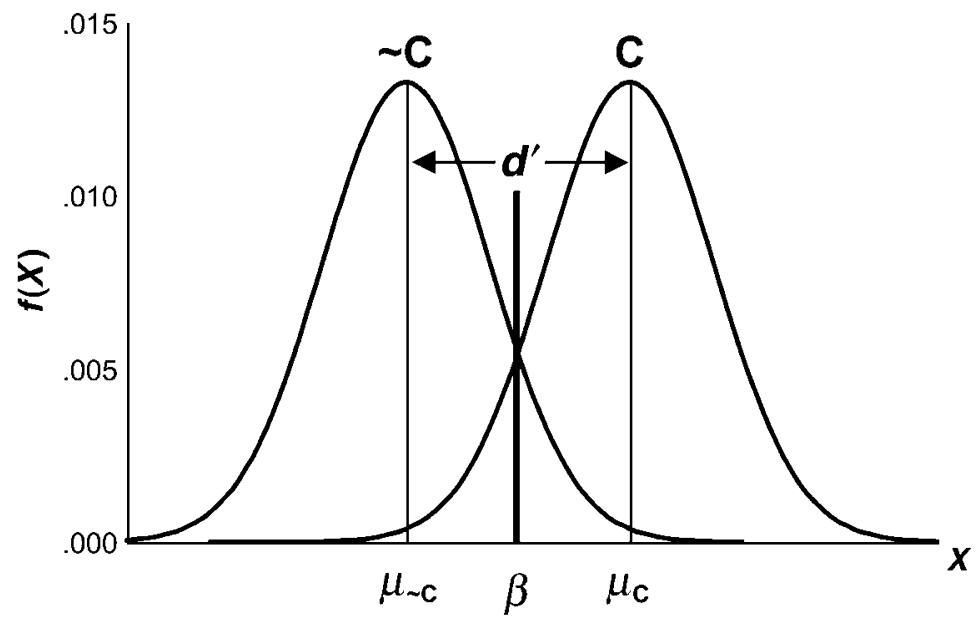

Figure 6. Schematic of the observer's decision problem. On the left is the probability distribution of sensory values $(X)$ resulting from noise $(\sim C)$, and on the right is the distribution resulting from signal plus noise $(C)$. The height of the probability function is denoted by $f(X)$. The means of the distribution are $\mu_{\sim \mathrm{C}}$ and $\mu_{\mathrm{C}} \cdot d^{\prime}$ is the difference between the means in standard deviation units. $\beta$ represents the criterion. 


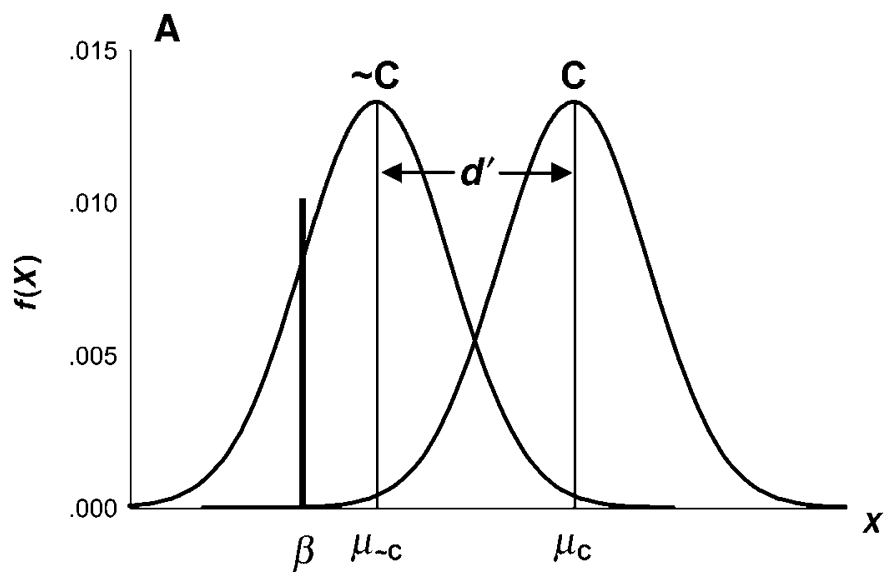

B

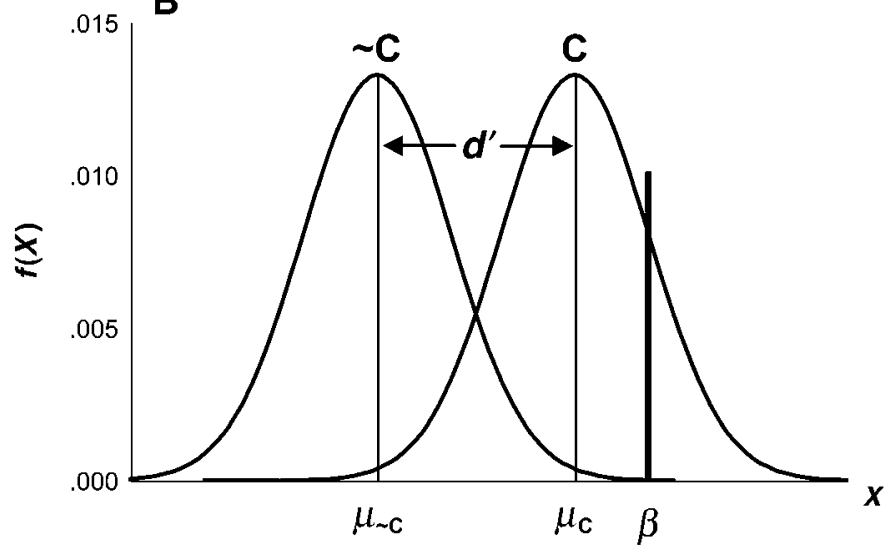

Figure 7. Two placements of the response criterion $\beta$ : (A) liberal, (B) conservative.

contingent and contingent matrices, respectively]. As density increases, the criterion moves along the ROC from conservative to liberal and as contingency increases sensitivity increases, moving from a low $d^{\prime}(.25)$ to a higher $d^{\prime}(1.90){ }^{2}$
This separation of sensitivity and criterion is displayed in a different way in Figure 10. Mean $d^{\prime}$ for each outcome density is plotted for the two contingencies in Figure 10A. For each of the noncontingent and contingent matrices, $d^{\prime}$ is constant across density. ${ }^{3} \beta$ as indexed by the place-

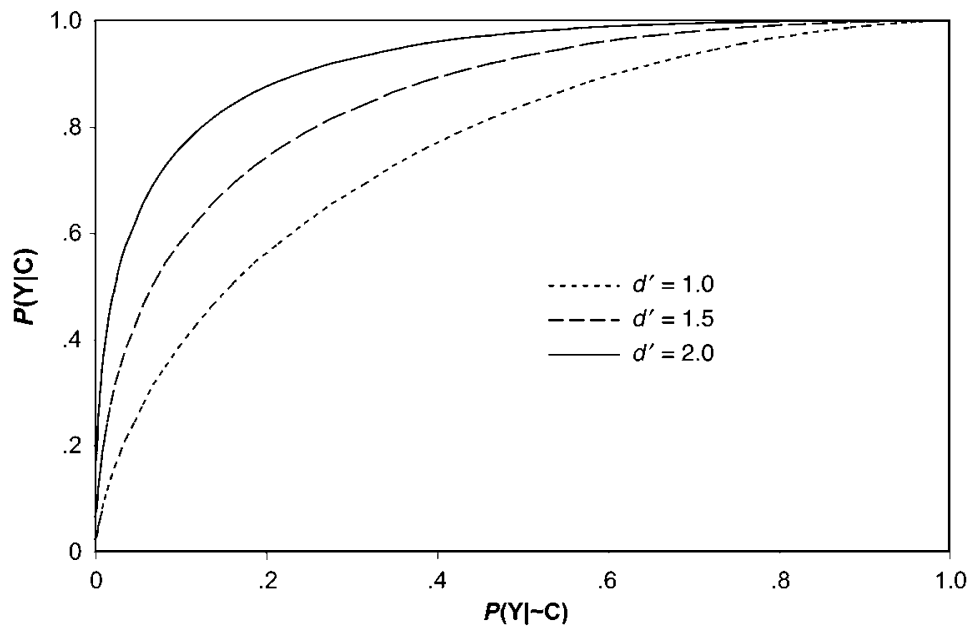

Figure 8. ROCs for three different signal values $\left(d^{\prime}=1.0,1.5\right.$, and 2.0). $P(\mathbf{Y} \mid \mathrm{C})$ is plotted as a function of $P(\mathrm{Y} \mid \sim \mathrm{C})$. 


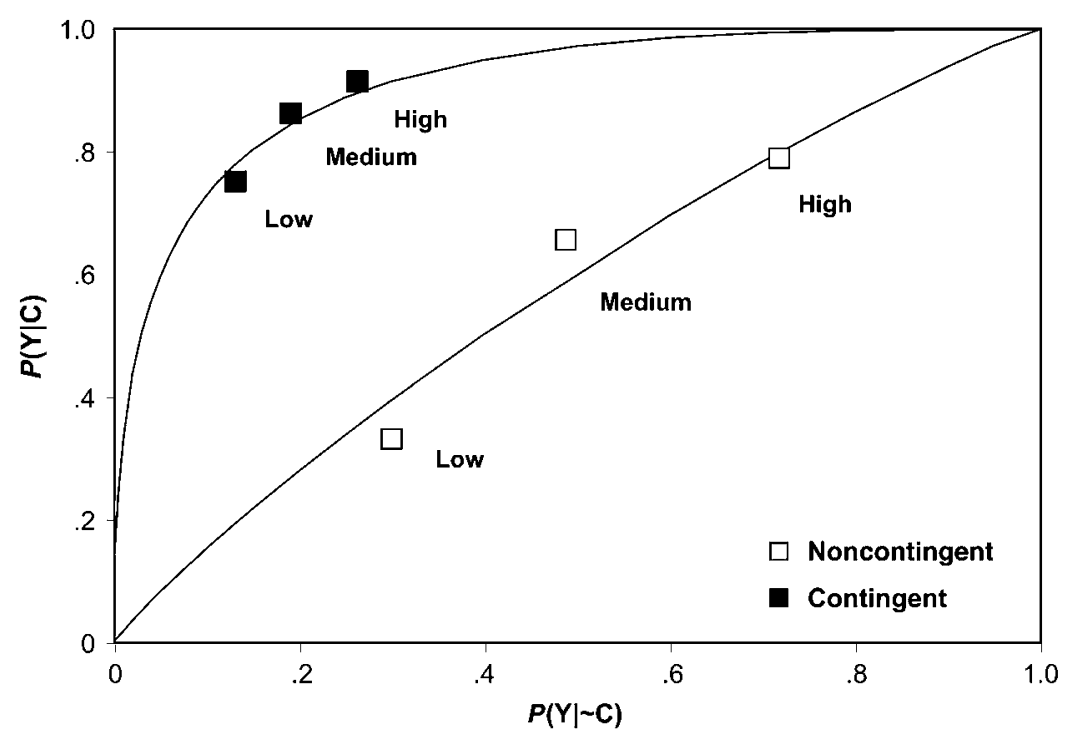

Figure 9. ROCs based on 60 trials. The lower set of three points was generated from the three noncontingent matrices, and the upper set of three points was generated from the three contingent matrices. The labels Low, Medium, and High identify the outcome-density matrix. Each curve is the best-fitting ROC to the three data points.

ment of the criterion relative to the mean of $\sim \mathrm{C}$ (i.e., $z(\mathrm{Y} \mid \sim \mathrm{C})$; see Equation $5 \mathrm{~B})$ is shown in Figure 10B. For each of the contingencies, $\beta$ decreases as density increases, indicating an increasing bias toward responding Y. Figure 10A (which plots $d^{\prime}$ ) and Figure 4 (which plots $\left.\Delta P_{\mathrm{PRED}}\right)$ show the same pattern of results. This, of course, is not surprising since both measures are based on the same conditional probabilities: $\Delta P_{\mathrm{PRED}}$ on the probabilities and $d^{\prime}$ on the $z$-transformation of the probabilities. What is striking is the relationship of the data displayed in Figure 10B (which plots $\beta$ ) to the data displayed in Figure 2 (which plots ratings). Figure 2 shows that increasing density results in higher ratings and Figure 10B shows that increasing density results in an increasing tendency to predict that the outcome will occur. The SDT analysis reveals that ratings reflect criterion location $(\beta)$, not sensitivity to contingency $\left(d^{\prime}\right)$.

Thus far, we have focused our SDT analysis on data from all 60 trials. In Figure 11, we plot ROCs based on the first 30 and last 30 trials. ${ }^{4}$ The curves are the best-fitting ROCs to the six data points ( 3 densities $\times 2$ trial blocks), and again the deviations are not significant [Pearson $\chi^{2}(5)=2.614, p>.05$, and $\chi^{2}(5)=2.128, p>.05$, for the noncontingent and contingent ROCs, respectively]. The $d^{\prime}$ values, .33 and 1.95 for the noncontingent and contingent curves, respectively, are similar to those based on all 60 trials. For the noncontingent matrices, the points are more spread out for the last 30 trials than for the first 30 trials, suggesting that the effect of density on the criterion increases over trials.

\section{DISCUSSION}

We have replicated the outcome-density effect with regard to ratings obtained after a block of trials: Ratings of the relationship between a cue and an outcome increase as the probability of the outcome increases even though the contingency does not change. In contrast, the $\Delta P_{\mathrm{PRED}}$ analysis indicated that the probability of the outcome has no effect on the observer's perception of the contingency as measured by trial-by-trial prediction responses. Thus, there appears to be a dissociation between the ratings and the prediction responses. The SDT analysis of the data provides an explanation of this apparent dissociation. While outcome density does not affect sensitivity to the contingency (i.e., $d^{\prime}$ ), it does affect the observer's willingness to predict that the outcome will occur (i.e., $\beta$ ). The overall probability of predicting that the outcome will occur increases with $P(\mathrm{O})$, and the ratings are a reflection of this tendency to predict $Y$ with increasing values of $P(\mathrm{O})$. The availability of two parameters in SDT allows the isolation of response criterion from sensitivity and reveals that the frequently observed density effect in contingency ratings is a criterion effect. Observers simply respond Y more often as $P(\mathrm{O})$ increases, and their ratings reflect this bias. Sensitivity to the actual size of the contingency (i.e., to $\Delta P_{\mathrm{O}}$ ) is not affected by outcome density.

\section{Density Effects and Signal Detection Theory}

Outcome- and cue-density effects are phenomena of contingency judgment tasks. If our suggestion that the contingency judgment task may be fruitfully analyzed as a signal detection task is correct, we would expect that signal detection tasks also should display such density effects. Because we were interested in the outcome-density effect, we varied $P(\mathrm{O})$ - that is, the column totals in Table 1A. As we increased $P(\mathrm{O})$, our observers increased $P(\mathrm{Y})$ - that is, the column totals in Table 1B. Varying $P(\mathrm{O})$ in a contingency assessment task is analogous to 

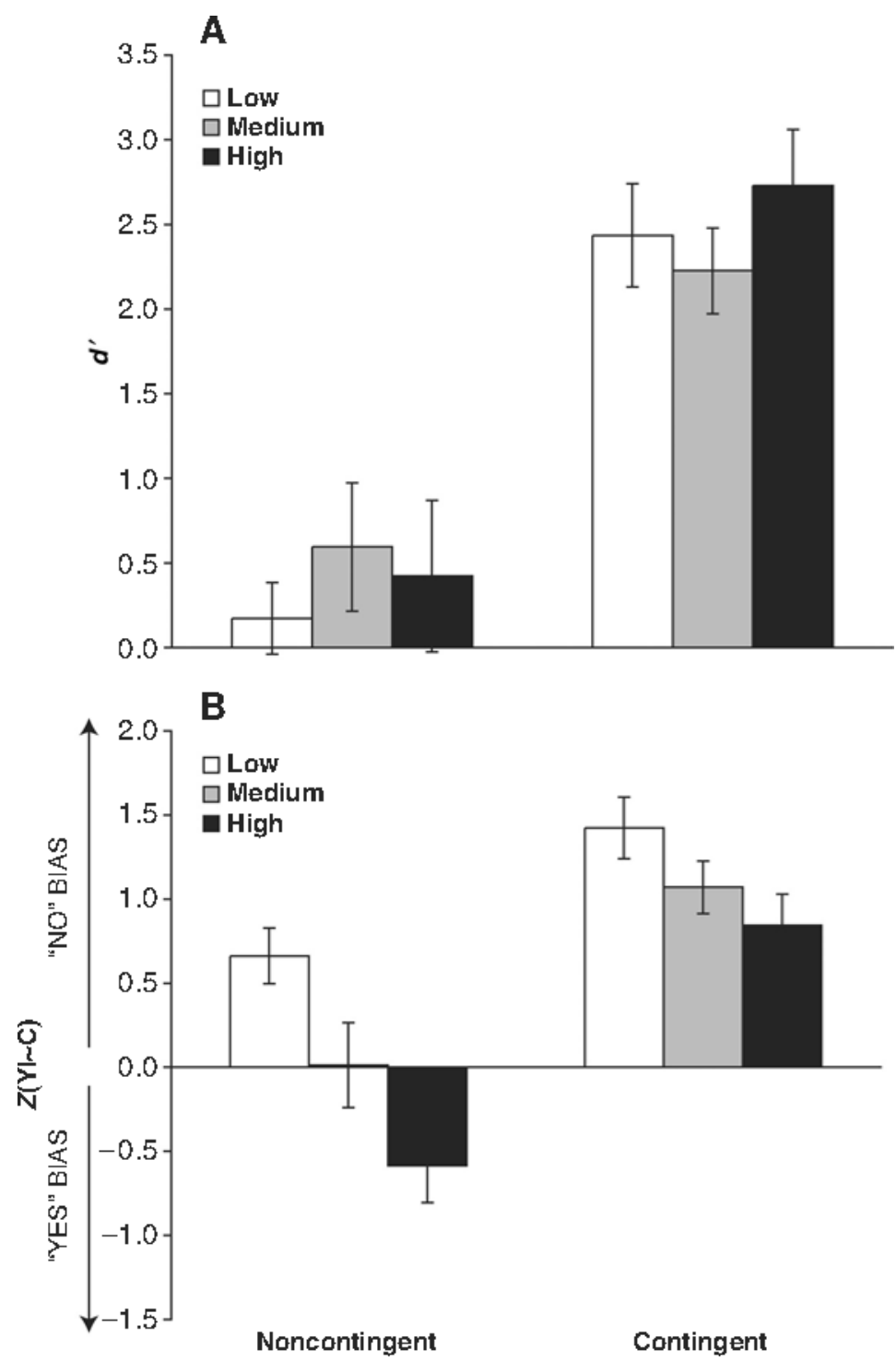

Figure 10. (A) $d^{\prime}$ for each outcome density (low, medium, and high). The values for the three noncontingent matrices $\left(\Delta P_{O}=0\right)$ are on the left, and the values for the three contingent matrices $\left(\Delta P_{O}=.467\right)$ are on the right. (B) $\beta$ for each outcome density (low, medium, and high). The values for the three noncontingent matrices $\left(\Delta P_{O}=0\right)$ are on the left, and the values for the three contingent matrices $\left(\Delta P_{O}=.467\right)$ are on the right. Note that positive values of $\beta$ indicate a bias for "NO" (conservative criterion) and negative values of $\beta$ indicate a bias for "YES" (liberal criterion).

varying feedback in a signal detection experiment - that is, the column totals in Table 1C. Typically, the column totals are not manipulated in a signal detection experiment. Rather, the feedback in a signal detection experiment is veridical-a correct response is identified as correct, and an incorrect response is identified as incorrect. Thus, the column totals in Table 1C would be determined by the observer's responses. However, at least one experiment did manipulate the veridicality of feedback. Kinchla and Atkinson (1964) varied the column totals in Table $1 \mathrm{C}$ by providing misinformation in their ex- periment. Specifically, on some of the no-signal $(\sim \mathrm{C})$ trials, the observer was told that a signal had occurred. This manipulation effectively increases $P(\mathrm{O})$. Kinchla and Atkinson found that this false information increased both $P(\mathrm{Y} \mid \mathrm{C})$ and $P(\mathrm{Y} \mid \sim \mathrm{C})$, compared with a condition where the feedback was veridical, but that $d^{\prime}$ was unaffected. Increasing $P(\mathrm{O})$ results in increases in $P(\mathrm{Y})$ both in contingency assessment and in signal detection.

In most signal detection experiments, it is signal probability, $P(\mathrm{C})$, that is varied, not feedback, $P(\mathrm{O})$. In such studies, the usual finding is that $P(\mathrm{Y})$ increases with 


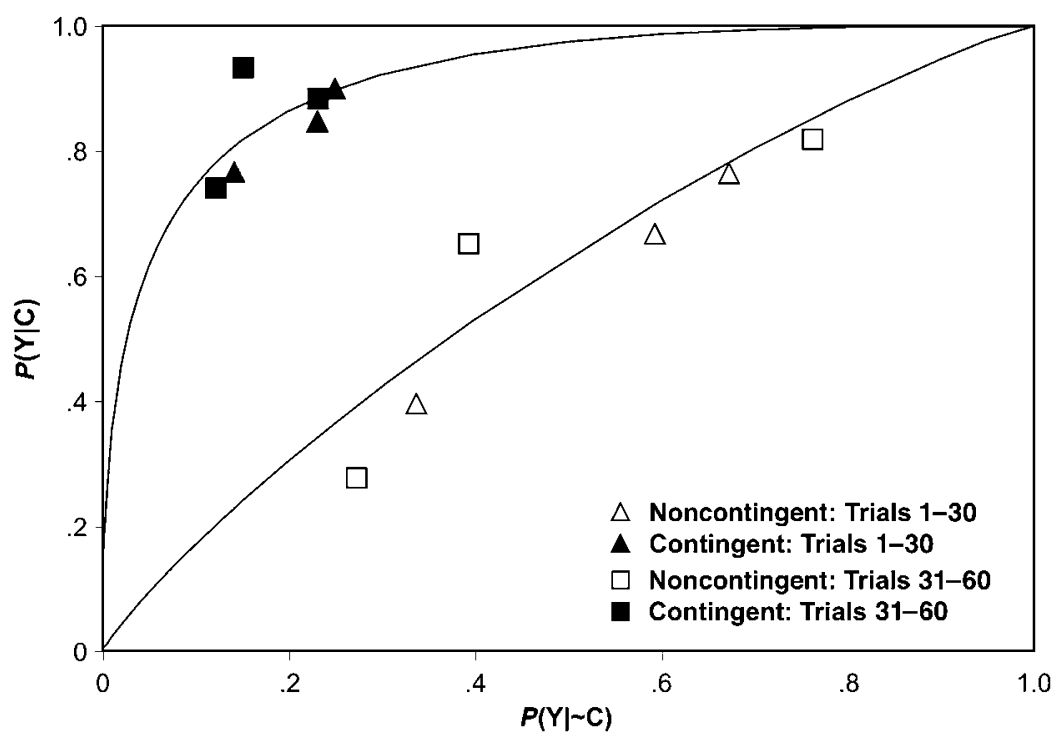

Figure 11. ROCs based on two blocks of 30 trials. The lower set of six points was generated from the three noncontingent matrices, and the upper set of six points was generated from the three contingent matrices. Each curve is the best-fitting ROC to the six data points.

$P(\mathrm{C})$ and that $d^{\prime}$ remains invariant (e.g., Tanner, Haller, \& Atkinson, 1967; Tanner, Rauk, \& Atkinson, 1970; see also Green \& Swets, 1966). The analogous contingency experiment would vary cue probability. As was noted earlier, Allan and Jenkins (1983) did vary $P(C)$ in their Experiment 3, and they reported that ratings increased with $P(\mathrm{C})$ - a cue-density effect (see Figure 1). Allan and Jenkins, however, did not collect prediction responses. On the basis of the present data, we would expect that an experiment that varied $P(\mathrm{C})$ and collected prediction responses would reveal that the cue-density effect is also located in the criterion parameter. That is, variations in $P(C)$ would affect $\beta$ but not $d^{\prime}$.

Our analysis suggests that while ratings are informative with regard to the observer's decision strategy, they might not provide an accurate picture of the observer's sensitivity to the contingency. In fact, there is a growing literature indicating that obtained ratings depend very much on the nature of the rating question (see Vadillo, Miller, \& Matute, 2005). We suggest that if an SDT analysis were applied to the prediction responses in these studies, one might find that $d^{\prime}$ did not vary across conditions but that $\beta$ did, and moreover that the ratings are a manifestation of $\beta$.

\section{A Contingency Judgment Payoff Matrix}

If judgments of contingency relationships are affected by $\beta$, it follows that other manipulations that affect $\beta$ should affect such judgments. It has been shown that a payoff matrix is a very effective way to manipulate $\beta$ (e.g., Healy \& Kubovy, 1978; see Green \& Swets, 1966). A payoff matrix is a depiction of the costs and benefits resulting from making correct and incorrect predictions.
Consider the payoff matrix in Table 4A. The observer earns 10 points for responding correctly to a signal, $(\mathrm{Y} \mid \mathrm{C})$, and earns only 1 point for responding correctly to noise, $(\sim \mathrm{Y} \mid \sim \mathrm{C})$. Moreover, the observer loses 5 points for responding incorrectly to a signal, $(\sim \mathrm{Y} \mid \mathrm{C})$, and loses only 2 points for responding incorrectly to noise, $(\mathrm{Y} \mid \sim \mathrm{C})$. This payoff matrix would encourage making $Y$ responses. In contrast, the payoff matrix in Table 4B would encourage making $\sim$ Y responses. Many signal detection studies have shown that while the payoff matrix affects $\beta$, it leaves $d^{\prime}$ untouched (see Green \& Swets, 1966, pp. 88-91). On the basis of a signal detection analysis, payoff matrix manipulations should affect judgments of contingency.

\section{Signal Detection, Contingency Judgment, and Subjective Values}

The astute reader will have noticed that the nature of the decision axis in Figure 6 needs further discussion. When describing Figure 6 earlier, we identified $X$ as representing the subjective values elicited by the physical values $\sim \mathrm{C}$ and $\mathrm{C}$. When the physical dimension is a sensory one, it is easy to relate the random variable $X$ to that physical dimension. For example, if the task is auditory detection, then the physical dimension is intensity, and $X$ is loudness. SDT has often been applied to the analysis of data where the translation of the physical to the subjective is not as straightforward. For example, in recognition memory experiments, the physical dimension is familiarity (old words vs. new words). The mapping is not as clear as with sensory dimensions. This is also the case in applying the SDT analysis to contingency data.

One solution to the dilemma has been provided by the likelihood ratio representation of the decision problem 
Table 4A

A Payoff Matrix That Would Encourage Y Responses

\begin{tabular}{rrrr}
\hline & $\mathrm{Y}$ & $\sim \mathrm{Y}$ \\
\hline $\mathrm{C}$ & 10 & -5 \\
$\mathrm{C}$ & -2 & 1 \\
\hline
\end{tabular}

Note-The numbers in the cells represent points gained or lost for each of the four cue-outcome combinations.

Table 4B

A Payoff Matrix That Would Encourage $\sim Y$ Responses

\begin{tabular}{rrr}
\hline & $\mathrm{Y}$ & $\sim \mathrm{Y}$ \\
\hline $\mathrm{C}$ & 1 & -2 \\
$\mathrm{C}$ & -5 & 10 \\
\hline
\end{tabular}

Note-The numbers in the cells represent points gained or lost for each of the four cue-outcome combinations.

(see Wickens, 2002, pp. 150-171, for a more detailed and mathematical discussion than can be presented here). The likelihood ratio representation assumes that the operative psychological variable is an odds ratio associated with the trial event. The odds ratio is equal to the likelihood that the event was drawn from the $\mathrm{C}$ distribution, $\mathrm{L}(\mathrm{C})$, divided by the likelihood that it was drawn from the $\sim \mathrm{C}$ distribution, $\mathrm{L}(\sim \mathrm{C})$. Thus, the $X$ axis in Figure 6 may in fact represent values of a likelihood ratio and the vertical axis is the probability that a particular value of $X$ resulted from $\sim \mathrm{C}$ or $\mathrm{C}$. If the likelihood ratio, $[\mathrm{L}(\mathrm{C})] /$ $[\mathrm{L}(\sim \mathrm{C})]$, is greater than a criterion value $\beta$, the response is $\mathrm{Y}$, and if the likelihood ratio is less than $\beta$, the response is $\sim$ Y. As a psychological model, the likelihood representation provides for a description of how decisions might be made in a contingency judgment task. The observer learns about the likelihood of the outcome on $\sim \mathrm{C}$ and $\mathrm{C}$ trials. On each trial, the observer refers to these likelihood distributions and decides how likely the outcome is. The comparison of likelihoods eliminates the need for a subjective sensory dimension. As Wickens has shown, under many circumstances the predictions for the likelihood representation are identical to those of the more traditional representation. They are identical for the equal variance normal distributions used in the analyses of our data.

Other representations for Figure 6 have also been suggested. For example, White and Wixted (1999; Wixted \& Gaitan, 2002) provided an account for delayed matchingto-sample in the pigeon where $X$ represents a sense of prior occurrence (analogous to familiarity in human recognition memory) on a given trial. In their model, the organism's behavior is governed by its history of reinforcement for making one response or the other under similar conditions in the past. The organism learns the relative probabilities of reinforcement for values of $X$ on the sense-of-prior-occurrence axis, and the response is based on these learned reinforcer ratios.

Our goal in the present paper is not to argue for the specifics of a particular detection model, but rather for the utility of applying such models to the analysis of contingency data. We think that this approach is useful in that it unconfounds perceptual and cognitive processing (encoding) from decision processes.

\section{Signal Detection, Contingency Judgment, and Associative Learning}

Early analyses of human judgment of contingency were rule-based models; they represented observers as intuitive statisticians who extract contingency information by applying a rule to integrate probabilities or frequencies of events (e.g., they computed $\Delta P_{\mathrm{O}}$ ) (see Allan, 1993). We have discussed the utility of an alternative approach involving the application of SDT to contingency judgment tasks. This application provides a novel interpretation of the outcome-density effect. Further research is needed to determine whether the SDT conceptualization of the contingency task can account for many of the other phenomena that are well established in the contingency assessment literature.

There are, of course, a variety of models that have been applied to explain the results of contingency judgments by humans. In something of a tour de force, Dickinson, Shanks, and Evenden (1984) provided an associative analysis of contingency judgments. Since then, there have been tremendous developments attesting to the value of applying simple, well-formulated models of learning, typically obtained from studies of the acquisition of conditional responses in nonhuman animals, to understanding the behavior of humans judging contingencies (Dickinson, 2001). These models trace their paternity to the Rescorla-Wagner formulation of learning (Rescorla \& Wagner, 1972; Wagner \& Rescorla, 1972). Although there are nonassociative models of contingency judgment, some have argued that the most fruitful analyses are those initially developed to explain results obtained from the nonhuman animal learning laboratory (see Allan \& Tangen, 2005). It is intriguing to consider the possibility that SDT is not simply another alternative to such associative interpretations of contingency judgments, but rather that SDT is a useful way of evaluating the acquisition of associations, by nonhuman animals as well as by humans.

In a previous publication, we (Siegel \& Allan, 1996, p. 319) suggested that there were similarities between SDT and the Rescorla-Wagner model: "Signal detection theory has been found to be applicable to many areas in addition to the limited domain in which it was first developed; that is, it has become a way to think about issues in areas other than psychophysics (see Swets, 1973, 1988). Similarly, the Rescorla-Wagner model has provided a basis for thinking about issues in areas other than Pavlovian conditioning. There are only a precious few such inspirational contributions in experimental psychology" (Siegel \& Allan, 1996, p. 319). Perhaps there is a synergistic relationship between these two particular "inspirational models."

\section{REFERENCES}

Allan, L. G. (1980). A note on measurement of contingency between two binary variables in judgment tasks. Bulletin of the Psychonomic Society, 15, 147-149. 
Allan, L. G. (1993). Human contingency judgments: Rule-based or associative? Psychological Bulletin, 114, 435-448.

Allan, L. G., \& JenKINS, H. M. (1983). The effect of representations of binary variables on judgment of influence. Learning \& Motivation, 14, 381-405.

ALLAN, L. G., \& Siegel, S. (2002). A signal detection analysis of the placebo effect. Evaluation \& the Health Professions, 25, 410-420.

Allan, L. G., \& TANGEN, J. M. (2005). Judging relationships between events: How do we do it? Canadian Journal of Experimental Psychology, 59, 22-27.

ALlan, L. G., TANGEN, J. M., Wood, R., \& ShaH, T. (2003). Temporal contiguity and contingency judgments: A Pavlovian analogue. Integrative Physiological \& Behavioral Science, 38, 214-229.

BLOUGH, D. S. (2004). Reaction time signatures of discrimination processes: Differential effects of stimulus similarity and incentive. Learning \& Behavior, 32, 157-172.

Collins, D. J., \& Shanks, D. R. (2002). Momentary and integrative response strategies in causal judgment. Memory \& Cognition, 30, 1138-1147.

DICKINSON, A. (2001). Causal learning: An associative analysis. Quarterly Journal of Experimental Psychology, 54B, 3-25.

DiCKInSON, A., Shanks, D. R., \& Evenden, J. (1984). Judgment of actoutcome contingency: The role of selective attribution. Quarterly Journal of Experimental Psychology, 36A, 29-50.

GREEN, D. M., \& Swets, J. A. (1966). Signal detection theory and psychophysics. New York: Wiley.

Healy, A. F., \& Kubovy, M. (1978). The effects of payoffs and prior probabilities on indices of performance and cutoff location in recognition memory. Memory \& Cognition, 6, 544-553.

KinCHLA, R. A., \& ATKINSON, R. C. (1964). The effect of false-information feedback upon psychophysical judgments. Psychonomic Science, 1, 317-318.

Macmillan, N. A., \& Creelman, C. D. (1991). Detection theory: A user's guide. Cambridge: Cambridge University Press.

Rescorla, R. A., \& Wagner, A. R. (1972). A theory of Pavlovian conditioning: Variations in the effectiveness of reinforcement and nonreinforcement. In A. H. Black \& W. F. Prokasy (Eds.), Classical conditioning II: Current research and theory (pp. 64-99). New York: Appleton-Century-Crofts.

ShanKs, D. R. (1993). Human instrumental learning: A critical review of data and theory. British Journal of Psychology, 84, 319-354.

Shanks, D. R., HolyoAK, K. J., \& Medin, D. L. (EDS.) (1996). The psychology of learning and motivation: Vol. 34. Causal learning. San Diego: Academic Press.

Siegel, S., \& Allan, L. G. (1996). The widespread influence of the Rescorla-Wagner model. Psychonomic Bulletin \& Review, 3, 314-321.

SwETs, J. A. (1973). The relative operating characteristic in psychology. Science, 182, 990-1000.

SWETS, J. A. (1988). Measuring the accuracy of diagnostic systems. Science, 240, 1285-1293.

SWETS, J. A. (1996). Signal detection theory and ROC analysis in psychology and diagnostics: Collected papers. Hillsdale, NJ: Erlbaum.

Tangen, J. M., \& Allan, L. G. (2003). The relative effect of cue interaction. Quarterly Journal of Experimental Psychology, 56B, 279-300.

TANGEN, J. M., \& ALlan, L. G. (2004). Cue-interaction and judgments of causality. Memory \& Cognition, 32, 107-124.

Tanner, T. A., JR., Haller, R. W., \& AtKinson, R. C. (1967). Signal recognition as influenced by presentation schedules. Perception \& Psychophysics, 2, 349-358.
Tanner, T. A., JR., Rauk, J. A., \& Atkinson, R. C. (1970). Signal recognition as influenced by information feedback. Journal of Mathematical Psychology, 7, 259-274.

Vadillo, M. A., Miller, R. R., \& Matute, H. (2005). Causal and predictive-value judgments, but not predictions, are based on cueoutcome contingency. Learning \& Behavior, 33, 172-183.

WAGNER, A. R., \& Rescorla, R. A. (1972). Inhibition in Pavlovian conditioning: Application to a theory. In R. A. Boakes \& M. S. Halliday (Eds.), Inhibition and learning (pp. 301-336). London: Academic Press.

White, K. G., \& Wixted, J. T. (1999). Psychophysics of remembering. Journal of the Experimental Analysis of Behavior, 71, 91-113.

WICKENS, T. D. (2002). Elementary signal detection theory. New York: Oxford University Press.

WiXted, J. T., \& Gaitan, S. C. (2002). Cognitive theories as reinforcement history surrogates: The case of likelihood ratio models of human recognition memory. Animal Learning \& Behavior, 30, 289-305.

\section{NOTES}

1. We used a program made available by Thomas Wickens, at http:// ist-socrates.berkeley.edu/ twickens/sdt.htm, to fit the ROCs. The program also compares the observed points and the predicted values using the Pearson $\chi^{2}$ statistic (see Wickens, 2002, pp. 245-247).

2. SDT is a continuous model: A specified physical value can result in an infinite number of subjective values with different probabilities In contrast, there are discrete models that map a constant physical value onto a few subjective values. One such model, the double high-threshold (DHT) model (see Macmillan \& Creelman, 1991), is of interest because of its relationship to $\Delta P$. According to DHT, the sensitivity parameter $\delta$ is

$$
\delta=P(\mathrm{Y} \mid \mathrm{C})-P(\mathrm{Y} \mid \sim \mathrm{C})=\frac{\mathrm{A}}{\mathrm{A}+\mathrm{B}}-\frac{\mathrm{C}}{\mathrm{C}+\mathrm{D}} .
$$

A comparison of Equation 7 with Equation 3 reveals that $\delta$ and $\Delta P_{\mathrm{PRED}}$ have identical calculations with regard to the $2 \times 2$ matrix. Both are equal to the difference between two conditional probabilities.

The DHT ROC is

$$
P(\mathrm{Y} \mid \mathrm{C})=P(\mathrm{Y} \mid \sim \mathrm{C})+\delta .
$$

For DHT, $P(\mathrm{Y} \mid \mathrm{C})$ is a linear function of $P(\mathrm{Y} \mid \sim \mathrm{C})$ with unit slope and intercept $\delta$. Our data cannot distinguish between DHT and SDT because the criterion spread is not large enough to discriminate between the SDT curvilinear ROC and the DHT linear ROC. However, the psychophysical literature has ample data to reject DHT (see Macmillan \& Creelman, 1991).

3. For the three noncontingent matrices, the $\mathrm{C}$ and $\sim \mathrm{C}$ distributions in Figure 6 are congruent and $d^{\prime}$ should be zero. Figure 10A indicates that $d^{\prime}$ is consistently greater than zero, and the best-fitting ROC in Figure 9 is $d^{\prime}=.25$. To determine whether $d^{\prime}$ differs significantly from zero, we conducted one-sample $t$ tests to compare mean $d^{\prime}$ for each density to zero. For all three densities, the difference was nonsignificant $[t(19)=.82$, 1.58 , and $.95, p>.05$, for low, medium, and high densities, respectively]

4. Since we obtained ratings at the end of 20 trial blocks, we considered calculating $\Delta P_{\mathrm{PRED}}$ on the basis of 20 trial blocks as well. However, we decided on larger blocks because with only 20 trials, row frequencies of zero occasionally occurred, and $\Delta P_{\mathrm{PRED}}$ values could not be calculated. With 30 trials, all row frequencies for all observers were greater than zero. Moreover, estimates of $\Delta P_{\mathrm{PRED}}$ based on 30 trials are more stable than estimates based on fewer trials. 